\title{
ChATty B cells
}

Apart from mediating interneuronal communication, neurotransmitters can regulate immune responses. For example, acetylcholine binds to muscarinic or nicotinic receptors on macrophages to modulate cytokine expression. Unexpectedly, it was recently shown that choline acetyltransferase (ChAT), which generates acetylcholine, is

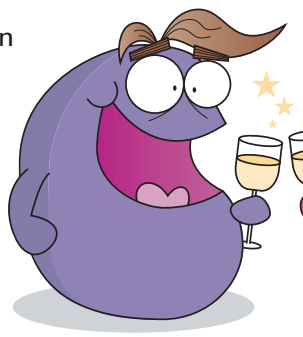
expressed not only by neurons but also by $\mathrm{CD}^{+} \mathrm{T}$ cells. Now, Reardon et al. further characterize ChAT expression in immune cells and report an anti-inflammatory role for $B$ cell-derived acetylcholine.

The authors observed that $\mathrm{CD} 4^{+} \mathrm{T}$ cells are not the only immune cells expressing ChAT. ChAT expression was identified in some splenic dendritic cells and macrophages, as well as in a substantial percentage of follicular and marginal zone B cells, lymph node and Peyer's patch B cells, and peritoneal B cells. Strikingly, ChAT was absent from thymocytes and immature $B$ cells, which led the authors to investigate the stimuli that induce its expression in mature lymphocytes.

ChAT expression could be induced following the activation of the B cell receptor and CD40, as well as by Toll-like receptor (TLR) ligands in splenic or peritoneal B cells. Notably, MYD88, which is a central adaptor protein in TLR signalling, was essential for this induction. Thus, B cells respond to microbial components by upregulating the expression of ChAT.

Consistently, ChAT expression was absent from fetal B cells but appeared in B cells after birth, suggesting a role for commensals. Indeed, the elimination of the intestinal microbiota through antibiotic treatment during weaning reduced the numbers of $\mathrm{ChAT}^{+} \mathrm{B}$ cells. Moreover, the induction of $\mathrm{ChAT}^{+} \mathrm{B}$ cells was more pronounced in mucosa-associated lymphoid tissues, where numerous microbial encounters occur.

Finally, microorganism-induced ChAT expression was shown to be transient, and - similarly to what is observed in neurons - acetylcholine generated by transiently expressed ChAT was released from B cells only after their stimulation with neurotransmitters, such as cholecystokinin. B cell-derived acetylcholine failed to suppress cytokine production or effector T cell responses in a mouse model of colitis. By contrast, local acetylcholine production by B cells was sufficient to inhibit neutrophil recruitment in the peritoneal cavity of mice following lipopolysaccharide injection. This was due to the impaired upregulation of adhesion molecules on endothelial cells as a result of acetylcholine-muscarinic receptor signalling.

Together, these findings indicate that B cells, and possibly other immune cells, may produce acetylcholine at sites of infection, where this neurotransmitter can then suppress neutrophil-mediated pathology.

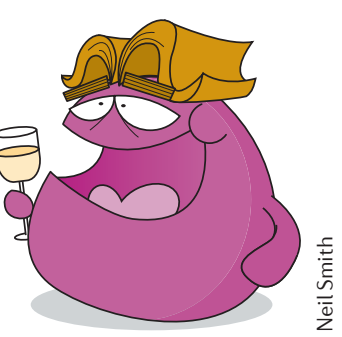

4

local

acetylcholine

production

by B cells

was sufficient

to inhibit

neutrophil

recruitment 